\title{
Wnt-RhoA Signaling Pathways in Fluoride-Treated Ameloblast-Lineage Cells
}

\author{
Kate Shusterman ${ }^{\mathrm{a}}$ Carolyn W. Gibson $^{\mathrm{a}}$ Yong Li ${ }^{\mathrm{a}}$ Melissa Healey ${ }^{\mathrm{a}}$ Li Peng $^{\mathrm{b}}$ \\ a Department of Anatomy and Cell Biology, School of Dental Medicine, University of Pennsylvania, \\ Philadelphia, Pa., USA; 'b State Key Laboratory of Oral Diseases, Sichuan University, Chengdu, PR China
}

\section{Key Words}

Ameloblast-like cells · RhoA pathway · Signal transduction ·

Wnt pathway

\begin{abstract}
This study examined the effect of sodium fluoride (NaF) on the Wnt and RhoA signaling pathways in murine ameloblastlineage cells (ALCs) to better understand the developmental mechanisms of dental fluorosis. Wnt and Rho pathway activities were investigated when ALCs were treated with 1.5 $\mathrm{mM} \mathrm{NaF}$, dickkopf-related protein-1 (Dkk-1), secreted frizzled related-protein-2 (sFRP-2), $\beta$-catenin siRNA dominant negative RhoA (RhoA ${ }^{\mathrm{DN}}$ ) plasmid and Y-27632. Wnt pathway activity was investigated via RT-PCR, Western blot and Topflash luciferase assay. The activity of the RhoA pathway was analyzed via Rho pull-down assay and immunoprecipitation. The differentiation of ALCs was analyzed by alkaline phosphatase assay. Western blot and Topflash luciferase assay results verified that both the Wnt and Rho pathways were upregulated by $1.5 \mathrm{~mm} \mathrm{NaF}$. Wnt was discovered to be located upstream from the Rho pathway, as confirmed by treatment with Wnt pathway cell receptor inhibitors Dkk-1 and sFRP-2, leading to a decrease in RhoA and ROCK activity. Inhibition
\end{abstract}

of the Rho pathway with RhoA $\mathrm{DN}^{\mathrm{DN}}$ plasmid and Y-27632 caused upregulation of Wnt pathway activity which could be further increased by $1.5 \mathrm{~mm} \mathrm{NaF}$. The increased Wnt pathway activity was found to negatively regulate ALC differentiation. These data suggest that fluoride could induce the cross-talk between Wnt and RhoA signaling pathways, and these responses are predicted to contribute to the development of enamel fluorosis.

(c) 2014 S. Karger AG, Basel

\begin{tabular}{ll}
\hline Abbreviations used in this paper \\
\hline ALCs & ameloblast-lineage cells \\
ALP & alkaline phosphatase \\
Dkk-1 & dickkopf-related protein-1 \\
LEF/TCF & lymphoid enhancer DNA-binding factor 1/ \\
& T cell-specific transcription factor \\
LRP5/6 & lipoprotein receptor-related proteins 5 and 6 \\
MEM & minimum essential medium \\
NaF & sodium fluoride \\
RhoADN & dominant negative RhoA \\
RLU & relative light units \\
sFRP-2 & secreted frizzled-related protein-2
\end{tabular}

\section{KARGER}

E-Mail karger@karger.com www.karger.com/cto
(C) 2014 S. Karger AG, Basel

$1422-6405 / 14 / 1993-0159 \$ 39.50 / 0$
Li Peng, $\mathrm{PhD}$

State Key Laboratory of Oral Diseases

West China Hospital of Stomatology, Sichuan University

3rd Section of Ren Min Nan Lu, Chengdu 610041 (PR China)

E-Mail pengscu@gmail.com 


\section{Introduction}

Fluoride has been the most effective treatment for the prevention of caries since the 1940s [Wong et al., 2011]. However, when consumed in excess during tooth development, fluoride will lead to a developmental disturbance of dental enamel in terms of enamel rod size, number, shape and quality of interaction, known as dental fluorosis [Eanes and Hailer, 1998]. Mild dental fluorosis is characterized by opaque white striations that run horizontally across the tooth [Alvarez et al., 2009]. In severe cases, the enamel pitting and porous regions increase in size, and discoloration of the enamel surface is seen [Bronckers et al., 2009]. When a transgenic mouse model expressing a dominant negative RhoA $\left(\mathrm{RhoA}^{\mathrm{DN}}\right)$ in secretory ameloblasts was generated, some similarities to enamel fluorotic defects in the cutting edge of incisors as well as cusps in the molar were observed [Li et al., 2011a]. Moreover, three independent transgenic mouse strains showed that fluoride-induced enamel alterations have similar changes in cell signaling mechanisms. We hypothesized that Wnt and RhoA pathways are implicated in fluoride-induced signal transduction in ameloblast-lineage cells (ALCs) as well as the enamel defects in Rho ${ }^{\text {DN }}$ transgenic mice [Peng et al., 2011].

Rho-mediated signaling potentially has an influential role in amelogenesis [Hatakeyama et al., 2009; Biz et al., 2010]. RhoA is one of the critical Rho GTPases that play a role in regulating the cytoskeleton as well as other cell activities [van Aelst and Symons, 2002; Burridge and Wennerberg, 2004]. RhoA is recognized as a molecular switch that normally cycles from the active GTP-bound form to the inactive GDP-bound form, and regulates downstream effector ROCK (Rho-associated protein kinase) leading to structural changes in the cytoskeleton of ameloblasts [Li et al., 2005].

The canonical Wnt pathway is $\beta$-catenin dependent and is activated when $\beta$-catenin enters the nucleus and interacts with lymphoid enhancer DNA-binding factor $1 / \mathrm{T}$ cell-specific transcription factor (LEF/TCF) transcription factors to promote downstream gene expression [Clevers, 2006]. Activation of canonical Wnt signaling is initiated by Wnt ligand binding to two receptors: frizzled andlipoprotein receptor-related proteins 5 and 6 (LRP5/6) [Nelson and Nusse, 2004]. Upon interaction, Disheveled inhibits the $\beta$-catenin degradation complex, which includes adenomatous polyposis coli, Axin proteins and glycogen synthase kinase-3 $\beta$. $\beta$-Catenin accumulates in the cytoplasm and nucleus, and nuclear $\beta$-catenin interacts with LEF/TCF to promote downstream gene expression [Clevers, 2006; MacDonald et al., 2009]. Various ap- proaches have indicated that the canonical Wnt pathway does not induce or even inhibit dental epithelial cell differentiation, but could maintain dental epithelial cells with normal biological character [Millar et al., 2003; Jarvinen et al., 2006; Chen et al., 2009; Lohi et al., 2010; Suomalainen and Thesleff, 2010].

Several studies have revealed cross-talk between Wnt and RhoA signaling pathways. Wnt3a, a canonical Wnt pathway family member, could stimulate osteoblastic differentiation, increase Chinese hamster ovary cell motility and induce neurite retraction through both $\beta$-catenin and RhoA/ROCK-dependent pathways [Kishida et al., 2004; Endo et al., 2005; Rossol-Allison et al., 2009]. RhoA/ ROCK could also act as the downstream factors of two noncanonical Wnt ligands, Wnt11 and Wnt5, to regulate convergence and extension movements of embryonic tissues in zebrafish embryos [Zhu et al., 2006] as well as dorsal cell elongation during zebrafish gastrulation [Marlow et al., 2002]. In contrast, RhoA signaling could impact on Wnt pathways. $\beta$-Catenin and Wnt5a were upregulated in enamel organs of Rho $\mathrm{A}^{\mathrm{DN}}$ transgenic mice, which expressed the Rho $\mathrm{A}^{\mathrm{DN}}$ transgene in ameloblasts [Peng et al., 2011]. This indicated that both canonical and noncanonical Wnt pathways were implicated in the formation of enamel defects in these mice.

The aim of this study was to better understand the interaction between the Wnt and Rho pathways when cells are treated with sodium fluoride $(\mathrm{NaF})$. This investigation will provide a better understanding of the mechanisms involved in fluoride-induced cell signal transduction.

\section{Materials and Methods}

\section{Reagents}

The following antibodies were sourced for this investigation: anti-RhoA, anti-GAPDH, anti- $\beta$-catenin, anti-p- $\beta$-catenin (Ser33/37/Thr41) from Cell Signaling Technology Inc. (Danvers, Mass., USA), anti-lamin B1 from Abcam Inc. (Cambridge, Mass., USA), anti-ROCK II from Santa Cruz Biotechnology Inc. (Santa Cruz, Calif., USA) and anti-phospho-MYPT1 (Thr850) from Millipore (Billerica, Mass., USA). The NE-PER Nuclear and Cytoplasmic Extraction Kit and Bradford Protein Concentration Assay Kit (Pierce, Rockford, Ill., USA), Protease Inhibitor Cocktail (Roche Diagnostics GmbH, Mannheim, Germany) and Phosphatase Inhibitor Cocktail (Sigma-Aldrich, St. Louis, Mo., USA) were also used. The inhibitors used were recombinant mouse secreted frizzled-related protein-2 (sFRP-2), recombinant mouse dickkopf-related protein-1 (Dkk-1; R\&D Systems, Minneapolis, Minn., USA). InSolution Y-27632 came from Calbiochem (La Jolla, Calif., USA). $\beta$-Catenin siRNA, control siRNA-A and siRNA transfection reagent were from Santa Cruz Biotechnology Inc. Topflash luciferase reporter plasmid (Upstate Biotechnology, Lake Placid, N.Y., USA), 
pRL-SV40 (Promega, Madison, Wis., USA), Lipofectamine Plus reagent (Invitrogen, Carlsbad, Calif., USA), Dual Luciferase Assay Kit (Promega, Madison, Wis., USA) and Rho Activation Assay Kit (Millipore) were all also obtained. StemTAG Alkaline Phosphatase Activity Assay Kit (Colormetric) came from Cell Biolabs Inc. (San Diego, Calif., USA).

\section{Cell Culture}

ALCs from a wild-type mouse [Takahashi et al., 2007] were grown in minimum essential medium (MEM) supplemented with $10 \%$ fetal bovine serum, $1 \%$ penicillin-streptomycin, $1 \%$ L-glutamine, $0.2 \mathrm{mM}$ calcium chloride and $10 \mathrm{ng} / \mathrm{ml}$ recombinant human epidermal growth factor (Sigma-Aldrich), and cells were passaged as needed. The medium was changed every other day, and fresh L-glutamine ( $1 \%$ of current medium volume) was added weekly.

\section{Treatment with Wnt Receptor Inhibitors}

When ALCs reached 70-80\% confluence, $0.1 \mu \mathrm{g} / \mathrm{ml}$ Dkk1, 0.15 $\mu \mathrm{g} / \mathrm{ml}$ SFRP2 or both were added to their respective groups. NaF was added to $1.5 \mathrm{mM} 6 \mathrm{~h}$ later. The RhoA and ROCK activities were then checked $4 \mathrm{~h}$ later, while cytoplasmic $\beta$-catenin and nuclear $\beta$-catenin levels were checked $8 \mathrm{~h}$ later. This experiment was repeated three times.

\section{Treatment with Rho $A^{D N}$ Plasmid and ROCK Inhibitor}

The RhoA ${ }^{\mathrm{DN}}$ (dominant negative RhoA) plasmid includes amelogenin regulatory sequences and partially blocks endogenous RhoA in ameloblasts by the N19 dominant negative mutation [Qiu et al., 1995; Li et al., 2011b]. Four micrograms of RhoA ${ }^{\mathrm{DN}}$ plasmid was transfected to the RhoA pathway blocking groups using $10 \mu \mathrm{l}$ of Lipofectamine 2000 in serum-free Opti-MEM (Invitrogen). Medium was replaced by MEM with 10\% FBS 6 h later and $1.5 \mathrm{mM}$ $\mathrm{NaF}$ was added $24 \mathrm{~h}$ after the medium replacement. Y-27632 was provided to ROCK-inhibited groups 6 h before $1.5 \mathrm{mM} \mathrm{NaF}$ was added. This experiment was repeated three times.

\section{Treatment of $\beta$-Catenin siRNA}

A total of $6.5 \mu$ of control siRNA or $\beta$-catenin siRNA were transfected into cells with $1 \mathrm{ml}$ of siRNA transfection reagent in siRNA transfection medium. After $6 \mathrm{~h}$ of treatment, $1 \mathrm{ml}$ of $4 \times$ normal growth medium was added to the cell plates. Cell samples in every treatment group were collected at different times depending on the target proteins being investigated. This experiment was repeated three times.

\section{Semiquantitative RT-PCR}

RNA was extracted from ALCs treated with or without $1.5 \mathrm{~mm}$ $\mathrm{NaF}$ for $24 \mathrm{~h}$. Two micrograms of total RNA was reverse transcribed and PCR was performed with a Veriti ${ }^{\mathrm{TM}}$ Thermal Cycler (Applied Biosystems, Foster City, Calif., USA) with Applied Biosystems PCR kit. The sequences of PCR primers were: $\beta$-catenin sense primer $5^{\prime}$-ACCTTTCAGATGCAGCGACT-3', antisense primer $5^{\prime}$-GTCGTGGAATAGCACCCTGT-3'; GAPDH sense primer $5^{\prime}$-TCACCACCATGGAGAAGGC-3', antisense primer $5^{\prime}$-GCTAAGCAGTTGGTGGTGCA-3'. The thermocycling profile was $8 \mathrm{~min}$ at $95^{\circ} \mathrm{C}$ for denaturing, $30 \mathrm{~s}$ at $95^{\circ} \mathrm{C}, 30 \mathrm{~s}$ at $58^{\circ} \mathrm{C}$, $45 \mathrm{~s}$ at $72^{\circ} \mathrm{C}$ for 30 cycles, with $10 \mathrm{~min}$ extension at $72^{\circ} \mathrm{C}$. PCR products were separated by electrophoresis on $1.5 \%$ agarose gels. $\beta$-Catenin band intensities were normalized against GAPDH as a control. This experiment was repeated three times.

Wnt-RhoA Pathways in ALCs

\section{Western Blot}

ALCs in each group were incubated on ice with protein lysis buffer (10 mM Tris pH 7.6, $100 \mathrm{~mm} \mathrm{NaCl}, 2$ mM EDTA, 0.5\% CHAPS) containing protease inhibitor cocktail and $1 \%$ phosphatase inhibitor cocktail. Protein concentrations were measured with Bradford Protein Concentration Assay according to the manufacturer's instructions. The NE-PER Nuclear and Cytoplasmic Extraction Kit was used according to the manufacturer's recommendations if extraction of separate cytoplasmic and nuclear protein fractions was necessary. In this experiment, cytoplasmic and nuclear $\beta$-catenin were extracted when ALCs were treated with Wnt receptor inhibitors. Total $\beta$-catenin and phosphorylated $\beta$-catenin expressions were investigated when the RhoA pathway was blocked. For each sample, $30 \mu \mathrm{g}$ of protein were loaded onto $4-20 \%$ gels (Thermo Fisher Scientific, Rockford, Ill., USA) followed by electrophoresis and transfer to polyvinylidene fluoride membranes using $300 \mathrm{~mA}$ for $2 \mathrm{~h}$. Membranes were blocked with 5\% nonfat milk for $2 \mathrm{~h}$ at room temperature and incubated with primary antibodies at $4^{\circ} \mathrm{C}$ overnight. After rinsing, membranes were incubated with HRP-conjugated secondary antibodies (1:5,000 dilution) at room temperature for $2 \mathrm{~h}$. Immunoreactive proteins were visualized by incubating membranes with SuperSignal West Femto Thermo Maximum Sensitivity Substrate (Thermo Scientific, Waltham, Mass., USA). Target proteins were detected using antibody for RhoA, GAPDH, $\beta$-catenin, $\mathrm{p}-\beta$-catenin (Ser33/37/Thr41), ROCK II and phospho-MYPT1 (Thr850; 1:1,000 dilution). This experiment was repeated three times.

\section{Topflash Dual-Luciferase Reporter Assays}

ALCs were plated in 24-well plates. According to the manufacturer's protocol, $1 \mu \mathrm{g}$ of Topflash (or Fopflash, which harbors mutant TCF-binding sites) luciferase reporter plasmid and $20 \mathrm{ng}$ of pRL-SV40 (RENILLA luciferase driven by the SV40 promoter) were transfected with the Lipofectamine Plus reagent. Topflash or Fopflash was transferred along with RhoA ${ }^{\mathrm{DN}}$ plasmid, control siRNA or $\beta$-catenin siRNA. After $24 \mathrm{~h}$, cells were treated with 1.5 $\mathrm{mM} \mathrm{NaF}$ for $8 \mathrm{~h}$. Luciferase activity was evaluated using the dual luciferase assay kit. Transcriptional activity was measured as relative light units (RLU), a ratio of firefly luciferase activity to Renilla activity. Each treatment was carried out in duplicate and the experiment was repeated three times.

\section{RhoA Activity Assay}

Fifteen microliters of Rho Assay Reagent slurry (Millipore) were added to each cell extract centrifuge tube. The reaction mixtures were incubated for $45 \mathrm{~min}$ at $4^{\circ} \mathrm{C}$ with gentle agitation followed by brief centrifugation $\left(10 \mathrm{~s}, 14,000 \mathrm{~g}, 4^{\circ} \mathrm{C}\right)$. After incubation, the supernatant was removed and the remaining beads were washed three times with $\mathrm{Mg}^{2+}$ Lysis/Wash Buffer (Millipore). The amount of immunoprecipitated GTP-RhoA was analyzed by immunoblot. The RhoA activity was expressed as the intensity of the GTP-RhoA band normalized by total RhoA. This experiment was repeated three times.

\section{Immunoprecipitation Analysis of ROCK II Activity}

Twenty-five micrograms of protein ALC lysate was added to 100 $\mu \mathrm{l}$ of prediluted protein $\mathrm{G}$ beads solution. The mixed solution was rotated on a shaker at $4^{\circ} \mathrm{C}$ for $10 \mathrm{~min}$ and centrifuged for $20 \mathrm{~s}$ at $3,000 \mathrm{rpm}$. Supernatant was collected and incubated with $0.5 \mu \mathrm{l}$ anti-ROCK II for $30 \mathrm{~min}$ while rotating on a shaker at $4^{\circ} \mathrm{C}$. Fresh 100 $\mu \mathrm{l}$ prediluted protein $\mathrm{G}$ beads solution was added and tubes were 


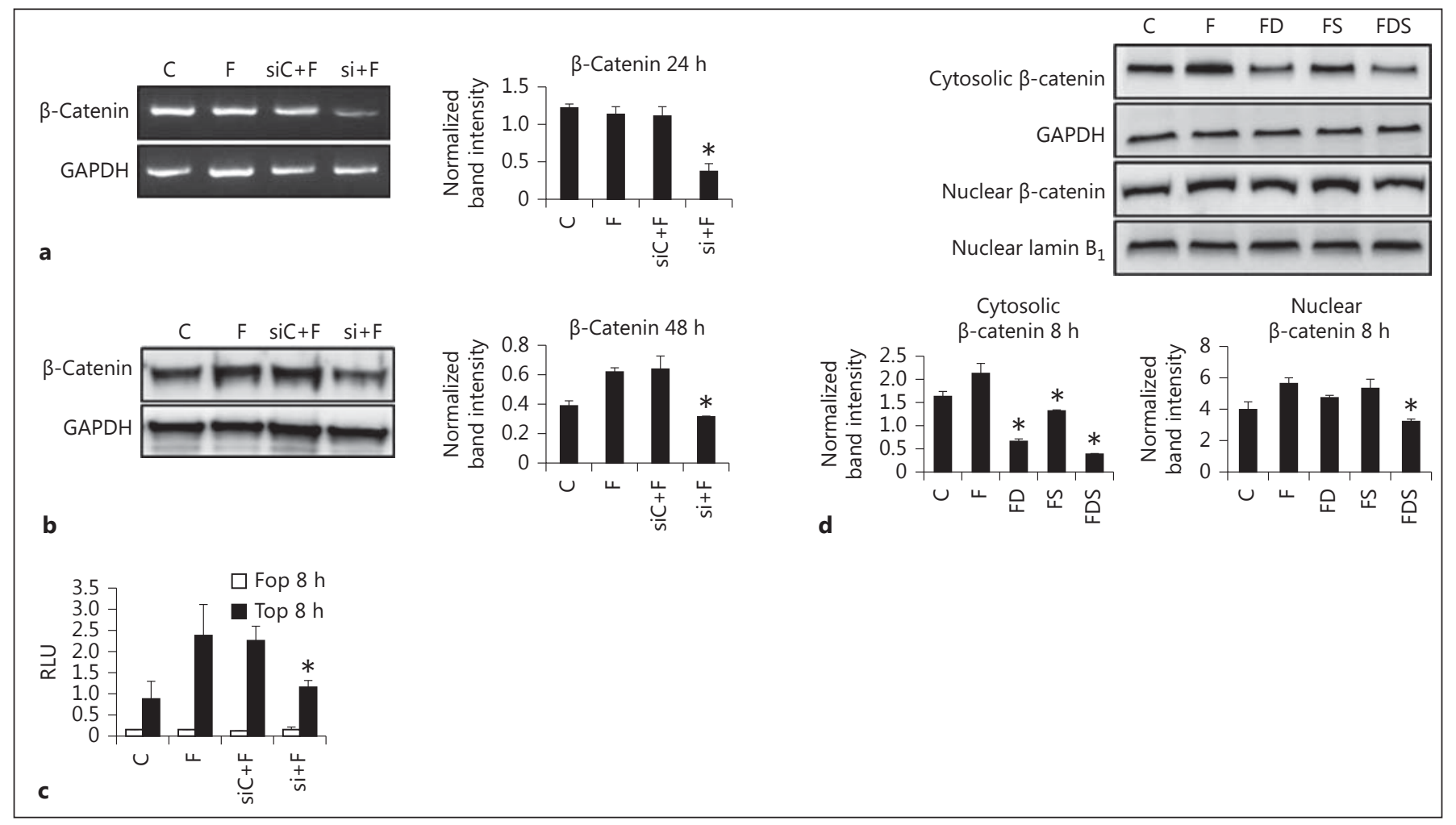

Fig. 1. Wnt pathway upregulation by $\mathrm{NaF}$ showed an increase of $\beta$-catenin protein expression and Topflash activity. Both Wnt pathway cell receptor inhibitors and $\beta$-catenin siRNA could inhibit the increase. a $\beta$-Catenin mRNA was downregulated by $\beta$-catenin siRNA. RT-PCR results showed that NaF did not alter $\beta$-catenin mRNA with or without control siRNA. However, $\beta$-catenin siRNA could decrease the $\beta$-catenin message significantly. b Western blot results showed that $\beta$-catenin was increased with $\mathrm{NaF}$ treatment and $\beta$-catenin siRNA could inhibit the increase. $\beta$-Catenin was normalized with GAPDH. c Topflash activity increased in $\mathrm{NaF}$ and the control siRNA $+\mathrm{NaF}$ group, but decreased in the $\beta$-catenin siRNA $+\mathrm{NaF}$-treated groups. Fopflash activity

shaken for $15 \mathrm{~min}$, and immediately centrifuged for $20 \mathrm{~s}$. Supernatant was discarded. To the immunoprecipitated ROCK beads, $20 \mu \mathrm{l}$ of lysis buffer, $1.5 \mu \mathrm{l}$ of $100 \mu \mathrm{M}$ ATP and $0.5 \mu \mathrm{l}$ of rMYTP1 was added. Sample tubes were incubated in a shaker $(300 \mathrm{rpm})$ at $37^{\circ} \mathrm{C}$ for $30 \mathrm{~min}$. Samples were boiled and run on SDS-PAGE. ROCK II activity was indicated by $\mathrm{p}-\mathrm{MYPT}-1$ band density normalized by that of total ROCK II. This experiment was repeated three times.

\section{Alkaline Phosphatase Assay}

Alkaline phosphatase assay (ALP) activity was quantified in ALCs harvested after $60 \mathrm{~h}$ in culture following the treatments described above, and the protein concentration was determined. Cell lysates were transferred to a 96-well plate and were processed for ALP activity using StemTAG Alkaline Phosphatase Activity Assay Kit according to the manufacturer's instruction. Absorbance of each well was read at $405 \mathrm{~nm}$ by using a Dynatech Laboratories MRX Microplate Reader. The activity was determined by absor- was not changed among all four groups. d Cytosolic and nuclear $\beta$-catenin protein levels with treatment of Wnt cell receptor inhibitors Dkk-1 and sFRP-2. Western blot showing the levels of cytosolic and nuclear $\beta$-catenin. Cytosolic $\beta$-catenin was normalized with GAPDH and nuclear $\beta$-catenin was normalized with lamin B1. Normalized band intensity of cytosolic and nuclear $\beta$-catenin showed that cytosolic $\beta$-catenin significantly decreased within all inhibitor groups and nuclear $\beta$-catenin decreased significantly in the FDS-treated group. $\mathrm{C}=\mathrm{Control} ; \mathrm{F}=\mathrm{NaF} ; \mathrm{FD}=$ $\mathrm{NaF}+$ Dkk1; FS $=\mathrm{NaF}+$ sFRP2; FDS $=\mathrm{NaF}+\mathrm{Dkk} 1+$ sFRP2; siC $+\mathrm{F}=$ control siRNA $+\mathrm{NaF} ; \mathrm{si}+\mathrm{F}=\beta$-catenin $\mathrm{siRNA}+\mathrm{NaF}$. $* \mathrm{p}<0.05$.

bance at 405 and normalized against the amount of protein in the reaction. Each treatment was carried out in duplicate and the experiment was repeated three times.

\section{Statistics}

Data are reported as means \pm SD. Statistical significance was assessed using Student's t tests for two groups or one-way analysis of variance and Tukey's post hoc test for more than two groups. Significance was defined as $\mathrm{p}<0.05$.

\section{Results}

The Wnt pathway core transcription factor $\beta$-catenin and $\mathrm{W}$ nt ligand receptors were investigated to confirm Wnt signal transduction in NaF-treated ALCs. ALCs 
Fig. 2. Rho $A^{D N}$ and $Y-27632$ could inhibit the activity of the Rho pathway in ALC treated with $\mathrm{NaF}$. Rho ${ }^{\mathrm{DN}}$ plasmid caused a significant decrease in RhoA activity $(\mathrm{p}<$ 0.05 ) and inhibition with Y-27632 led to a significant decrease in ROCK activity ( $\mathrm{p}<$ 0.05). $\mathrm{C}=$ Control; $\mathrm{F}=\mathrm{NaF} ; \mathrm{R}=\mathrm{RhoA}^{\mathrm{DN}}$; $\mathrm{FR}=\mathrm{NaF}+\mathrm{RhoA}^{\mathrm{DN}} ; \mathrm{Y}=\mathrm{Y}-27632 ; \mathrm{FY}=$ $\mathrm{NaF}+\mathrm{Y}-27632 .{ }^{*} \mathrm{p}<0.05$.

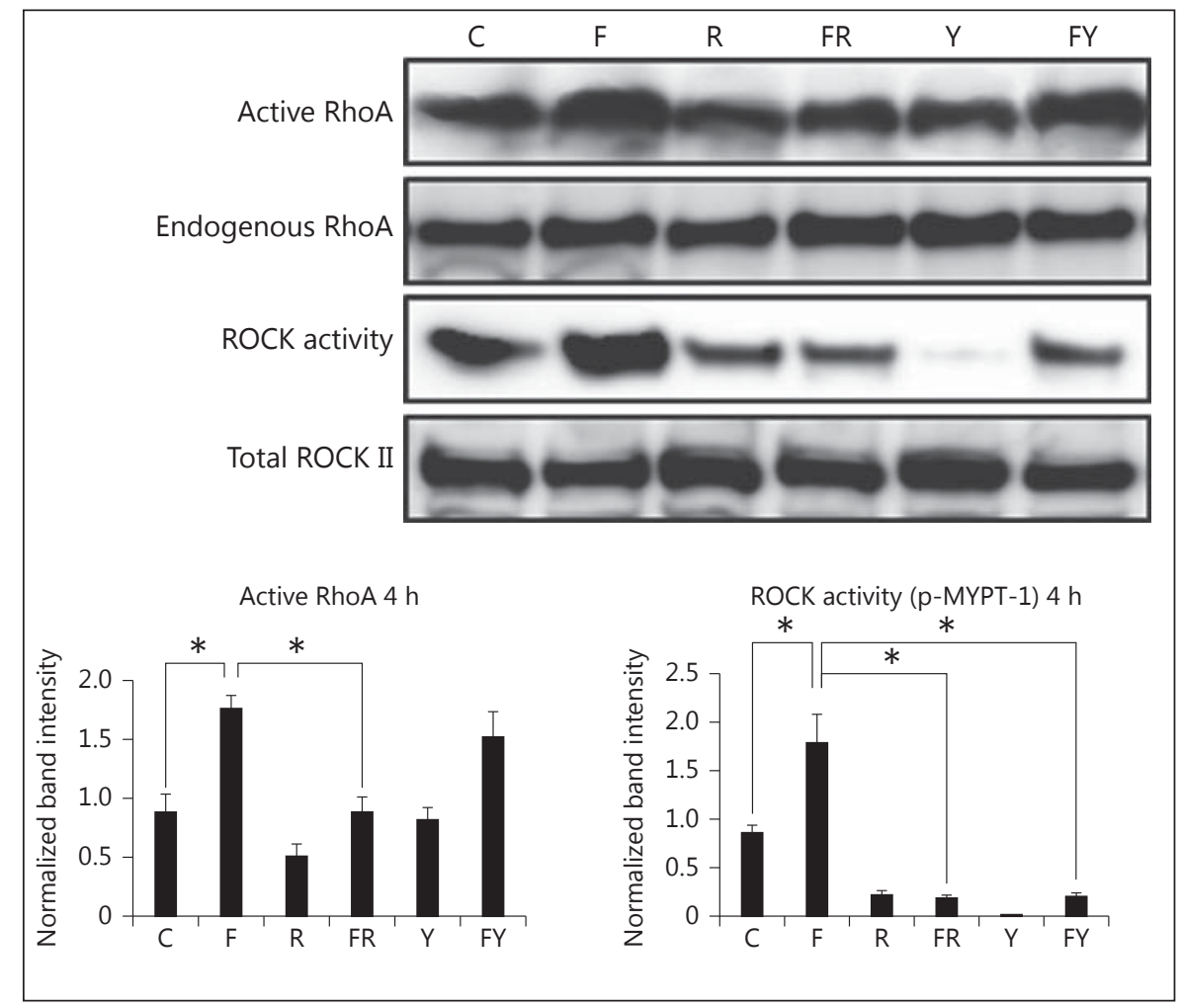

treated with control siRNA and $\beta$-catenin siRNA showed that the $\beta$-catenin siRNA downregulated both $\beta$-catenin mRNA and protein, while $\mathrm{NaF}$ could upregulate the $\beta$-catenin protein amount and downstream transcriptional activity (fig. 1a-c). Cells treated with $\beta$-catenin siRNA showed a significant decrease in Topflash activity $(\mathrm{p}<0.05)$ in comparison to the NaF plus control siRNA-treated groups (fig. 1c). The role of Wnt receptors in NaF-treated ALCs was also analyzed. ALCs treated with Wnt signal antagonists and $\mathrm{NaF}$ were analyzed using Western blot. Samples were collected $4 \mathrm{~h}$ following treatment with $1.5 \mathrm{mM} \mathrm{NaF}$. In order to confirm the efficiency of the Wnt cell receptor inhibitors Dkk-1 and sFRP-2, the levels of cytoplasmic and nuclear $\beta$-catenin were analyzed. Treatment with NaF led to high expression of both cytoplasmic and nuclear $\beta$-catenin. This upregulation of $\beta$-catenin could have occurred through either the conventional receptors, LRP5/6 or frizzled. Treatment with both of the Wnt cell receptor antagonists inhibited the Wnt signal, as demonstrated by a significant decrease in accumulation of cytoplasmic $\beta$-catenin and nuclear $\beta$-catenin ( $\mathrm{p}<0.05$; fig. $1 \mathrm{~d}$ ).

Rho pathway activity was also evaluated in NaF-treated ALCs. Rho pull-down and immunoprecipitation assays showed that $\mathrm{NaF}$ significantly upregulated activity of

Wnt-RhoA Pathways in ALCs the Rho pathway components RhoA and ROCK. Rho ${ }^{\text {DN }}$ and Y-27632 could inhibit the increase ( $<<0.05$; fig. 2 ).

In order to investigate how the Rho pathway activity was affected by the Wnt pathway, ALCs treated with Wnt signal antagonists and $\mathrm{NaF}$ were analyzed using a Rho pulldown assay and immunoprecipitation for activity of ROCK and Western blot. Treatment with Wnt pathway cell receptor inhibitors Dkk-1 and/or sFRP-2 caused a decrease in RhoA and ROCK activity. The greatest decrease in activity was seen when both Dkk-1 and sFRP-2 were used together (fig. 3a). With verification that siRNA downregulates the Wnt pathway, a Western blot was run for siRNA samples and incubated with RhoA antibodies to measure the amount of activated RhoA compared to total RhoA. NaF upregulated RhoA activity $(\mathrm{p}<0.05)$ in comparison to the control group. The level of RhoA activity significantly decreased with $\beta$-catenin siRNA treatment ( $<<0.05$; fig. $3 b$ ). In cells treated with $\beta$-catenin siRNA prior to treatment with $\mathrm{NaF}$, the Wnt pathway, and presumably $\beta$-catenin expression, will be downregulated. A significant recovery was seen in ALP activity for $\mathrm{NaF}+\beta$-catenin siRNA-treated cells. This result was statistically significant $(\mathrm{p}<0.05)$ in comparison to all other treatment groups (fig. 3c).

Finally, the role of the Rho pathway in relation to the canonical Wnt pathway was investigated. The expression 


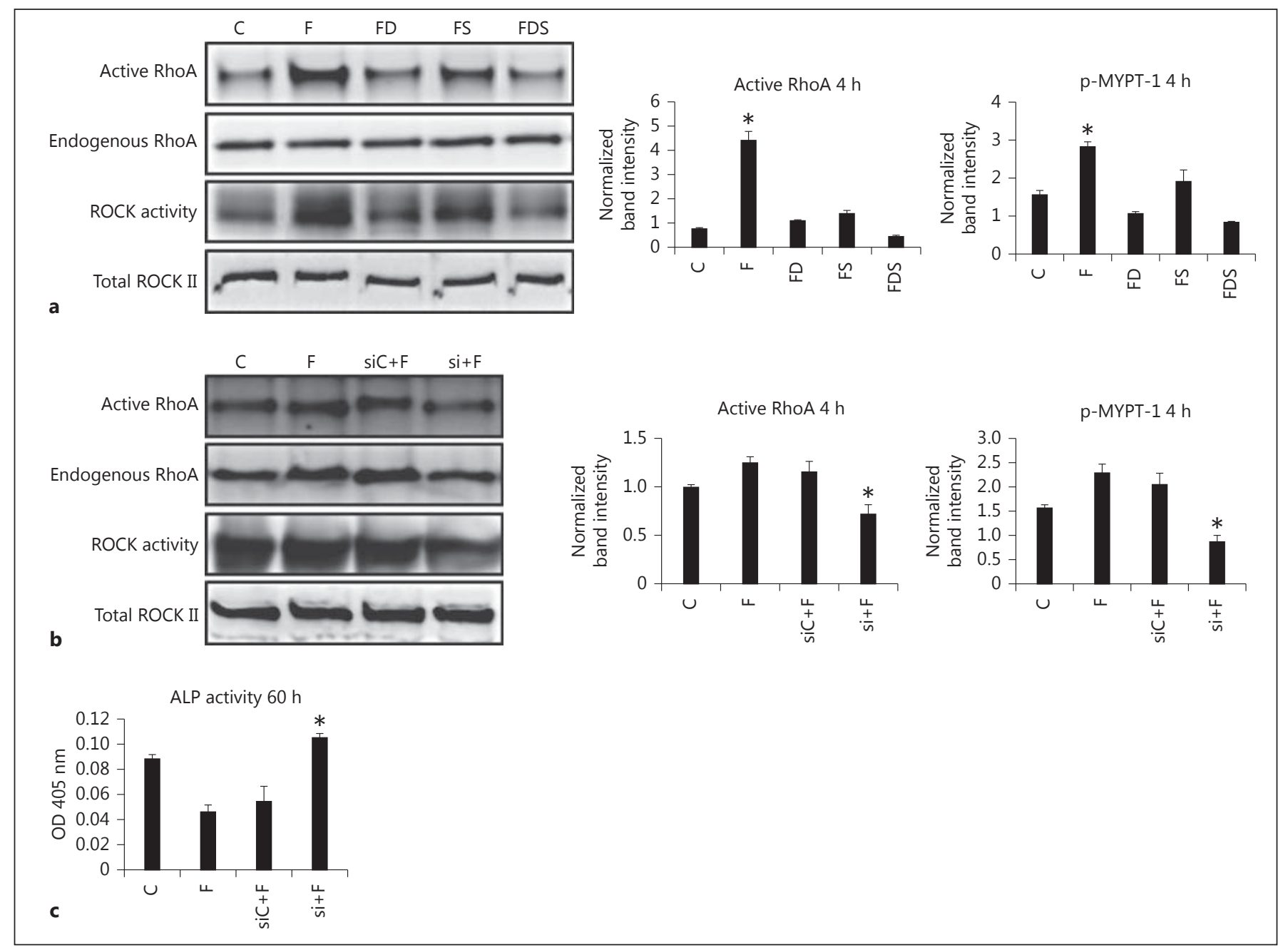

Fig. 3. RhoA and ROCK activities were decreased when the Wnt pathway was blocked by either receptor inhibitors or $\beta$-catenin siRNA. a RhoA and ROCK activities are downregulated by treatment with Wnt pathway cell receptor inhibitors Dkk-1 and sFRP2. Western blot showing activated RhoA and ROCK. Activated RhoA was normalized with total RhoA, and activated ROCK, measured by phosphorylated MYPT-1, was normalized with total ROCK, showing that both RhoA and ROCK activity were significantly increased by treatment with $\mathrm{NaF}(\mathrm{p}<0.05)$. A decrease in RhoA or ROCK activity is observed with treatment of receptor inhibitors Dkk-1, sFRP-2 or both (* $\mathrm{p}<0.05)$. b Activity of RhoA and ROCK decreased with $\beta$-catenin siRNA treatment. Western

of $\beta$-catenin and phosphorylated $\beta$-catenin proteins was investigated in ALCs treated by transfection with Rho ${ }^{\mathrm{DN}}$ or Y-27632. Blots were probed for $\beta$-catenin, $\mathrm{p}$ - $\beta$-catenin (Ser33/37/Thr41) and GAPDH. $\beta$-Catenin was increased by treatment with $\mathrm{NaF}$, Rho ${ }^{\mathrm{DN}}$ and Y-27632, while $\mathrm{p}-\beta$ catenin (Ser33/37/Thr41) was downregulated. The combination of $\mathrm{NaF}$ and $\mathrm{RhoA}^{\mathrm{DN}}$ significantly increased blot showing activated RhoA and ROCK. Activated RhoA was normalized with total RhoA, and activated ROCK, measured by phosphorylated MYPT-1, was normalized with total ROCK. RhoA and ROCK activity increased with treatment of $\mathrm{NaF}$ in comparison to the control group, and decreased with $\beta$-catenin siRNA treatment in comparison to the NaF-treated group $(* \mathrm{p}<0.05)$. c Alkaline phosphatase activity increased with $\beta$-catenin siRNA treatment. Alkaline phosphatase activity decreased in the NaF-treated group and increased significantly with $\beta$-catenin siRNA treatment $\left({ }^{*} \mathrm{p}<\right.$ 0.05). $\mathrm{C}=$ Control; $\mathrm{F}=\mathrm{NaF} ; \mathrm{FD}=\mathrm{NaF}+\mathrm{Dkk} 1 ; \mathrm{FS}=\mathrm{NaF}+\mathrm{sFRP} 2$; $\mathrm{FDS}=\mathrm{NaF}+\mathrm{Dkk} 1+\mathrm{sFRP} 2 ; \mathrm{siC}+\mathrm{F}=$ control siRNA $+\mathrm{NaF} ; \mathrm{si}+\mathrm{F}$ $=\beta$-catenin siRNA $+\mathrm{NaF}$.

$\beta$-catenin expression $(\mathrm{p}<0.05)$ in comparison to the $\mathrm{NaF}$ - or Rho $\mathrm{A}^{\mathrm{DN}}$-treated groups (fig. $4 \mathrm{a}, \mathrm{b}$ ). Transfection of the Rho pathway inhibitors Rho ${ }^{\mathrm{DN}}$ plasmid and treatment with Y-27632 showed an increase in Topflash luciferase activity compared to the control group. The addition of $\mathrm{NaF}$ to the RhoA ${ }^{\mathrm{DN}}$ - or Y-27632-treated group showed significant increases $(\mathrm{p}<0.05)$ in Topflash lucif- 
Fig. 4. Fluoride could upregulate the Wnt pathway independently of the RhoA pathway activity. The decreased RhoA pathway activity could also activate the Wnt pathway. a Canonical Wnt pathway was activated by treatment with $\mathrm{RhoA}^{\mathrm{DN}}$ and $\mathrm{Y}-27632$. Western blot showing expression of $\beta$-catenin and phosphorylated $\beta$-catenin protein with the treatment of NaF, RhoA ${ }^{\mathrm{DN}}$ and Y-27632. Blots were probed for $\beta$-catenin, $p$ - $\beta$-catenin (Ser33/37/Thr41) and GAPDH. b Normalized band intensity of $\beta$-catenin and phosphorylated $\beta$-catenin protein. $\beta$-Catenin was increased by treatment with $\mathrm{NaF}$, RhoA ${ }^{\mathrm{DN}}$ and Y-27632, while $\mathrm{p}$ - $\beta$-catenin (Ser33/37/Thr41) was downregulated. The combination of $\mathrm{NaF}$ and RhoA $^{\mathrm{DN}}$ significantly upregulate $\beta$-catenin $(\mathrm{p}<0.05)$. c Both RhoA ${ }^{\mathrm{DN}}$ - and Y-27632-treated groups had significantly higher luciferase activity $(\mathrm{p}<0.05)$ in comparison to the control group. The addition of $\mathrm{NaF}$ to the RhoA $\mathrm{DN}_{-}$treated group had significantly higher luciferase activity ( $\mathrm{p}<$ 0.05) in comparison to the NaF- or RhoA ${ }^{\mathrm{DN}}$-treated groups. d Treatment with NaF, RhoA ${ }^{\text {DN }}$ or Y-27632 could significantly decrease the ALP activity in ALCs $(p<0.05)$. The addition of $\mathrm{NaF}$ to the $\mathrm{RhoA}^{\mathrm{DN}}$ - or Y-27632-treated groups had the lowest $\operatorname{ALP}(\mathrm{p}<0.05) . \mathrm{C}=$ Control; $\mathrm{F}=$ $\mathrm{NaF} ; \mathrm{R}=\mathrm{RhoA}^{\mathrm{DN}} ; \mathrm{FR}=\mathrm{NaF}+\mathrm{RhoA}^{\mathrm{DN}}$; $\mathrm{Y}=\mathrm{Y}-27632 ; \mathrm{FY}=\mathrm{NaF}+\mathrm{Y}-27632{ }^{*} \mathrm{p}<0.05$.

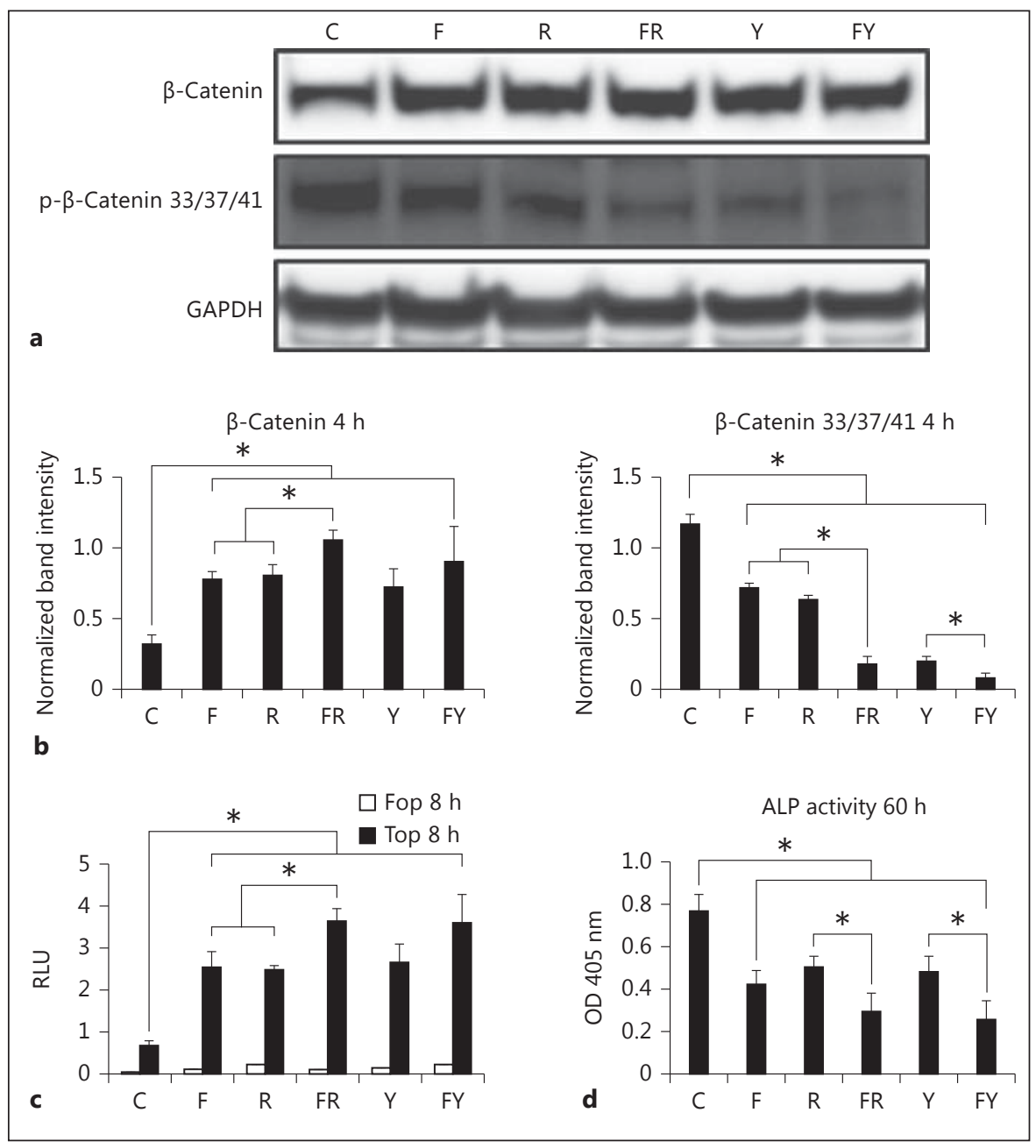

erase activity and, therefore, an upregulation of the Wnt pathway (fig. 4c). After $60 \mathrm{~h}$, ALC treated with $1.5 \mathrm{~mm}$ $\mathrm{NaF}$ showed a decrease in ALP activity. A statistically significant decrease in ALP activity was observed in groups treated with RhoA ${ }^{\mathrm{DN}}$ and Y-27632 ( $\left.\mathrm{p}<0.05\right)$ in comparison to the control group. The addition of $\mathrm{NaF}$ to the $\mathrm{RhoA}^{\mathrm{DN}}$ - or Y-27632-treated group showed significant decreases $(\mathrm{p}<0.05)$ in comparison with the Rho $\mathrm{A}^{\mathrm{DN}}$ - or Y-27632-treated groups (fig. 4d).

In summary, both Wnt and Rho pathways could be upregulated by NaF. The Wnt pathway was activated through its two receptors: frizzled and LRP 5/6. NaF could upregulate RhoA pathway, which was blocked by RhoA ${ }^{\text {DN }}$ and Y-27632. The RhoA pathway could also be activated by the Wnt pathway. Both Wnt pathway cell receptor inhibitors (DKK- 1 and sFRP-2) and $\beta$-catenin siRNA could block RhoA pathway activity. Decreased RhoA pathway activity could also activate the Wnt pathway (fig. 5).

\section{Discussion}

Previous in vivo studies done with Rho $\mathrm{A}^{\mathrm{DN}}$ transgenic mice that express RhoA with a dominant negative N19 mutation to decrease RhoA activity in ameloblasts [Qiu et al., 1995] concluded that interference with RhoA activity leads to surface defects in enamel [Li et al., 2011a]. Because the defects seen in RhoA ${ }^{\mathrm{DN}}$ transgenic mice were somewhat similar to those seen in cases of human enamel fluorosis, in the present study ALCs were treated with $\mathrm{NaF}$ to determine if the cultured cells respond through cell signaling pathways and how treatment affects the cross-talk between Wnt and Rho in ameloblast cells, leading to alterations in pathway component expression or activity.

The specific inhibitors used in this study were chosen because of their efficiency shown in previous experiments. Dkk-1 and sFRP-2 have been used in multiple 


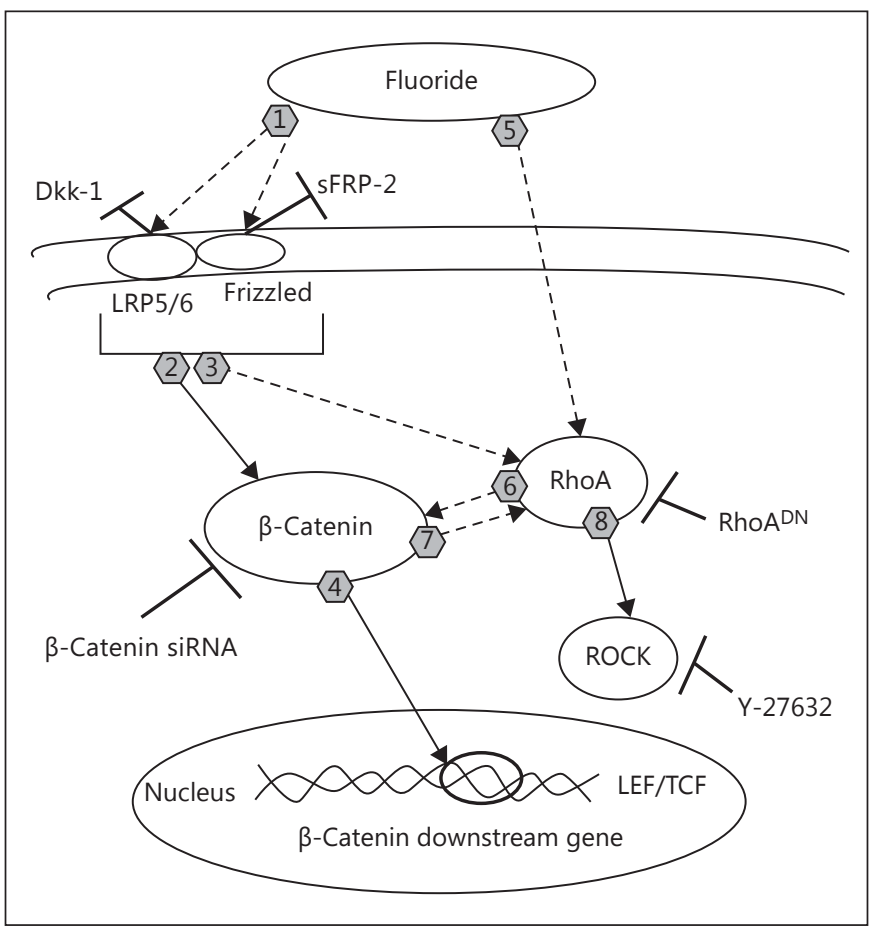

Fig. 5. Wnt and Rho pathways could be upregulated by NaF. Both Wnt pathway cell receptor inhibitors (Dkk-1 and sFRP-2) and $\beta$-catenin siRNA could block RhoA pathway activity. Decreased RhoA pathway activity could also activate the Wnt pathway. The whole cell signal pathway was divided into three main lines and two sublines. (1)(2)(4) The tree main lines $-\mathrm{NaF}$ could upregulate the Wnt pathway through its two receptors, frizzled and LRP5/6. (5) (8) $\mathrm{NaF}$ could upregulate the RhoA pathway, which could be blocked by RhoA ${ }^{\mathrm{DN}}$ and Y-27632. (1)(3)(7) The RhoA pathway could also be activated by the Wnt pathway. (7) (8) Two sublines - activity of RhoA and ROCK decreased with $\beta$-catenin siRNA treatment. (6) (4) The decreased RhoA pathway activity could also activate the Wnt pathway. Dashed lines indicate that the cell signal mechanism is as yet unclear; solid lines indicate that the mechanism has been reported.

studies to downregulate Wnt signaling through the inhibition of LRP5/6 and frizzled [Oshima et al., 2005; Liu et al., 2011]. Two efficient options for inhibition of $\beta$-catenin have been described, namely $\beta$-catenin siRNA and quercetin. Based on a literature review, siRNA has been used in ameloblast treatment [Duan et al., 2011; Otsu et al., 2011] and was determined to work efficiently. Also, Rho ${ }^{\mathrm{DN}}$ plasmid, which decreases intracellular RhoA activity, and Y-27632, a conventional inhibitor of ROCK, has been proven to inhibit RhoA and ROCK in ameloblasts [Qiu et al., 1995; Ishizaki et al., 2000]. The assays used measure different aspects of the pathways to avoid false results.
Since activation of Wnt signaling uncouples the degradation complex allowing an accumulation of $\beta$-catenin in the cytoplasm [Liu and Millar, 2010], a decrease in cytoplasmic $\beta$-catenin after the addition of Wnt pathway cell receptor inhibitors was expected. Although the sFRP2 group showed higher levels of cytoplasmic $\beta$-catenin than the groups treated with Dkk-1 or both inhibitors, an overall decrease in cytoplasmic $\beta$-catenin accumulation was seen when the Wnt pathway was downregulated. This verifies the effectiveness of the Wnt cell receptor inhibitors. The results for nuclear $\beta$-catenin did not seem to follow this trend. A decrease in all three inhibited groups (Dkk-1, sFRP-2 and both inhibitors) was expected; however, the only significant result was seen when both inhibitors were added together. This result could be caused by a translocation of accumulated $\beta$-catenin to the nucleus prior to the addition of inhibitors and collection of samples. It is apparent that nuclear $\beta$-catenin was not significantly influenced by either inhibitor when used alone; however, when used together the inhibitors proved to be efficient.

These experiments illustrated that $1.5 \mathrm{~mm} \mathrm{NaF}$ increased RhoA activity within $4 \mathrm{~h}$. The treatment of ameloblast cells with $\mathrm{NaF}$ led to a change in cellular activities through the inactivation of RhoGAP, which activates RhoA [Vincent et al., 1998; Li et al., 2005]. The addition of Wnt cell receptor antagonists Dkk-1 and sFRP-2 to cultured ALCs led to a decrease in RhoA activity, signifying that RhoA may be downstream of the Wnt pathway in these cells. The results of the RhoA activity assay help to explain how ROCK (measured by phosphorylated MYPT-1) is affected by Dkk-1 and sFRP-2. Again, an increase in activated ROCK was seen after treatment with $\mathrm{NaF}$. The Wnt pathway inhibitors caused a decrease in activated ROCK, thus further confirming that RhoA and its effector ROCK are downstream from the Wnt pathway. When the Wnt pathway was blocked, the Rho pathway was downregulated.

$\beta$-Catenin siRNA efficiency was verified by downregulation of $\beta$-catenin target expression in the nucleus using a Topflash gene report assay. Treatment with $\beta$-catenin siRNA led to a decrease in RhoA activity, again validating that Wnt and $\beta$-catenin are located upstream from RhoA. Lastly, treatment with $\beta$-catenin siRNA caused a significant restoration of ALP activity. This indicates that when the Wnt pathway is blocked, ALP activity increases. Since alkaline phosphatase activity is associated with cell differentiation [O'Gorman et al., 2012], high alkaline phosphatase activity in this case implies that the Wnt pathway may negatively regulate ALC differentiation. This is an 
expected result because Wnt signaling may act to restrict terminal differentiation in ameloblasts, and essentially arrest cells in a state of proliferation [Lohi et al., 2010].

$\mathrm{NaF}$ could activate the RhoA pathway in ALC within $4 \mathrm{~h}$ [Peng et al., 2011]. Therefore, treatment with Rho ${ }^{\mathrm{DN}}$ plasmid or Y-27632 in the presence of NaF causes a significant inhibition in RhoA or ROCK activity $4 \mathrm{~h}$ later. Blocking the Rho pathway may lead to an increase in Wnt pathway activity. Otsu et al. [2011] discovered that by blocking ROCK with Y-27632, $\beta$-catenin was able to translocate from the cell membrane to the nucleus. The Topflash gene report assay showed an increase in Wnt pathway activity in ALCs treated with $\mathrm{RhoA}^{\mathrm{DN}}$ or Y-27632. Western blot incubated with $\beta$-catenin antibody further confirmed the upregulation of $\beta$-catenin when the Rho pathway components RhoA and ROCK were inhibited. NaF could further upregulate Wnt pathway activity compared to Rho $\mathrm{A}^{\mathrm{DN}}$ treatment alone. However, treatment with Y-27632 and NaF did not cause a significant increase in Wnt activity, implying that $\mathrm{NaF}$ and ROCK may regulate Wnt signaling pathway activity through a pathway independent of Rho.

It is clear from previous experiments that treatment with $\mathrm{NaF}$ upregulates the Wnt pathway, as do inhibitors of the Rho pathway. Treatment with both of these upregulating factors presumably caused an increase in Wnt signaling, which led to a decrease in alkaline phosphatase activity. It is also possible that $\mathrm{NaF}$ can influence the differentiation of ALCs, as was discussed by Riksen et al. [2011], who confirmed that high concentrations of fluoride cause a reduction in ALP activity. In addition, Milan et al. [2001] validated that $0.09 \mathrm{M}(378 \mathrm{ppm})$ of fluoride inhibits ALP activity. Furthermore, previous studies have found a direct relationship between high ALP activity of ameloblasts and increased enamel mineralization [Gomez and Boyde, 1994]; thus, Wnt signaling may negatively regulate enamel mineralization.

It is most likely an apparent contradiction that fluoride results in both activation of Rho and Wnt signaling, and inhibits ALP production. Yet inhibition of Rho also inhibits ALP production. Thus, signals that oppositely affect Rho have the same effect on ALP activation. To the best of our knowledge, based on our data and that in the literature [Milan et al., 2001; Riksen et al., 2011], fluoride inhibits ALP activity. Even if fluoride results in both increased Rho activation and $\beta$-catenin stabilization, it does not mean that increased activation of Rho and Wnt signals lead to decreased ALP activity. Again, it is NaF, not RhoA, Wnt or $\beta$-catenin, that inhibits ALP activity. Figure $3 c$ shows that $\beta$-catenin stabilization had a negative effect on ALP activity, which is consistent with the effect of $\mathrm{NaF}$. Figure $4 \mathrm{~d}$ shows that RhoA activity had a similar trend to ALP activity. From this result, it indicated that increased RhoA may increase ALP activity. Fluoride could activate RhoA, Wnt and other unknown pathways. The common effect of fluoride is a decrease in ALP activity, and it is interesting to speculate the potential mechanisms. Firstly, as was mentioned above, ALP activity is decreased by common effects which include Wnt, Rho and other so far unknown signals. Next, it was verified that diminished $\beta$-catenin could upregulate ALP activity (fig. 1b, 3c). It is possible that activated Rho could upregulate ALP activity by decreasing Wnt secretion or $\beta$-catenin nuclear translocation. To clarify this, extensive experimentation has been performed.

The present study focused on NaF-induced cross-talk between the Wnt and Rho pathways to better understand the developmental mechanisms of enamel and enamel fluorosis. It is possible that enamel fluorosis can occur through the cross-talk of these pathways since both the Wnt and Rho pathways have increased activity when ALCs are treated with NaF. Figure 5 attempts to summarize these pathways in ALCs treated with NaF. This relationship is particularly important given that exposure to fluoride can occur through water, food and other common sources. Although the amount of fluoride ingested can be theoretically measured, there is a lack of knowledge in respect to the exact amount of fluoride that reaches the surface of ameloblasts. RhoA has been previously shown to have a significant role in the development of enamel, and high levels of Wnt signaling may lead to decreased enamel mineralization. These signaling pathways are involved in the development of other oral diseases, including ectopic teeth and taste papillae [Liu and Millar, 2010]; therefore, it is important to thoroughly understand how they function.

From the analysis of cells in vitro, it was proven that the Wnt and RhoA pathways play an important role in ALCs when treated with fluoride. The cross-talk between the Wnt and RhoA pathway, as well as its effects on the cell skeleton, is a promising and proven way to disclose the mechanisms of dental fluorosis.

\section{Acknowledgement}

We thank Dr. Toshihiro Sugiyama for his kind gift of ALCs. This work was supported by the National Institutes of Health by NIDCR grants R21-DE0176110 (C.W.G.) and the Cheung Family Scholarship (L.P.). 


\section{References}

Alvarez, J.A., K.M. Rezende, S.M. Marocho, F.B. Alves, P. Celiberti, A.L. Ciamponi (2009) Dental fluorosis: exposure, prevention and management. Med Oral Patol Oral Cir Bucal 14: E103-E107.

Biz, M.T., M.R. Marques, V.O. Crema, A.S Moriscot, M.F. dos Santos (2010) GTPases RhoA and Racl are important for amelogenin and DSPP expression during differentiation of ameloblasts and odontoblasts. Cell Tissue Res 340: 459-470.

Bronckers, A.L., D.M. Lyaruu, P.K. DenBesten (2009) The impact of fluoride on ameloblasts and the mechanisms of enamel fluorosis. J Dent Res 88: 877-893.

Burridge, K., K. Wennerberg (2004) Rho and Rac take center stage. Cell 116: 167-179.

Chen, J., Y. Lan, J.A. Baek, Y. Gao, R. Jiang (2009) Wnt/ $\beta$-catenin signaling plays an essential role in activation of odontogenic mesenchyme during early tooth development. Dev Biol 334: 174-185.

Clevers, H. (2006) Wnt/ $\beta$-catenin signaling in development and disease. Cell 127: 469-480.

Duan, X., Y. Mao, X. Wen, T. Yang, Y. Xue (2011) Excess fluoride interferes with chloride-channel-dependent endocytosis in ameloblasts. J Dent Res 90: 175-180.

Eanes, E.D., A.W. Hailer (1998) The effect of fluoride on the size and morphology of apatite crystals grown from physiologic solutions. Calcif Tissue Int 63: 250-257.

Endo, Y., V. Wolf, K. Muraiso, K. Kamijo, L. Soon, A. Uren, M. Barshishat-Kupper, J.S. Rubin (2005) Wnt-3a-dependent cell motility involves RhoA activation and is specifically regulated by Dishevelled-2. J Biol Chem 280: 777-786.

Gomez, S., A. Boyde (1994) Correlated alkaline phosphatase histochemistry and quantitative backscattered electron imaging in the study of rat incisor ameloblasts and enamel mineralization. Microsc Res Tech 29: 29-36.

Hatakeyama, J., S. Fukumoto, T. Nakamura, N. Haruyama, S. Suzuki, Y. Hatakeyama, L. Shum, C.W. Gibson, Y. Yamada, A.B. Kulkarni (2009) Synergistic roles of amelogenin and ameloblastin. J Dent Res 88: 318-322.

Ishizaki, T., M. Uehata, I. Tamechika, J. Keel, K. Nonomura, M. Maekawa, S. Narumiya (2000) Pharmacological properties of Y-27632, a specific inhibitor of Rho-associated kinases. Mol Pharmacol 57: 976-983.

Jarvinen, E., I. Salazar-Ciudad, W. Birchmeier, M.M. Taketo, J. Jernvall, I. Thesleff (2006) Continuous tooth generation in mouse is induced by activated epithelial Wnt $/ \beta$-catenin signaling. Proc Natl Acad Sci USA 103: 18627-18632.
Kishida, S., H. Yamamoto, A. Kikuchi (2004) Wnt-3a and Dvl induce neurite retraction by activating Rho-associated kinase. Mol Cell Biol 24: 4487-4501.

Li, Y., S. Decker, Z.A. Yuan, P.K. Denbesten, M.A. Aragon, K. Jordan-Sciutto, W.R. Abrams, J. Huh, C. McDonald, E. Chen, M. MacDougall, C.W. Gibson (2005) Effects of sodium fluoride on the actin cytoskeleton of murine ameloblasts. Arch Oral Biol 50: 681-688.

Li, Y., M.K. Pugach, M.A. Kuehl, L. Peng, J. Bouchard, S.Y. Hwang, C.W. Gibson (2011a) Dental enamel structure is altered by expression of dominant negative RhoA in ameloblasts. Cells Tissues Organs 194: 227-231.

Li, Y., M.K. Pugach, M.A. Kuehl, L. Peng, J. Bouchard, S.Y. Hwang, C.W. Gibson (2011b) Dental enamel structure is altered by expression of dominant negative RhoA in ameloblasts. Cells Tissues Organs 194: 227-231.

Liu, F., S.E. Millar (2010) Wnt/ $\beta$-catenin signaling in oral tissue development and disease. J Dent Res 89: 318-330.

Liu, N., S. Shi, M. Deng, L. Tang, G. Zhang, B. Ding, W. Liu, Y. Liu, H. Shi, L. Liu, Y. Jin (2011) High levels of $\beta$-catenin signaling reduce osteogenic differentiation of stem cells in inflammatory microenvironments through inhibition of the noncanonical Wnt pathway. J Bone Miner Res 26: 2082-2095.

Lohi, M., A.S. Tucker, P.T. Sharpe (2010) Expression of Axin 2 indicates a role for canonical Wnt signaling in development of the crown and root during pre- and postnatal tooth development. Dev Dyn 239: 160-167.

MacDonald, B.T., K. Tamai, X. He (2009) Wnt/ $\beta$ catenin signaling: components, mechanisms, and diseases. Dev Cell 17: 9-26.

Marlow, F., J. Topczewski, D. Sepich, L. SolnicaKrezel (2002) Zebrafish Rho kinase 2 acts downstream of Wnt11 to mediate cell polarity and effective convergence and extension movements. Curr Biol 12: 876-884.

Milan, A.M., R.J. Waddington, G. Embery (2001) Fluoride alters casein kinase II and alkaline phosphatase activity in vitro with potential implications for dentine mineralization. Arch Oral Biol 46: 343-351.

Millar, S.E., E. Koyama, S.T. Reddy, T. Andl, T. Gaddapara, R. Piddington, C.W. Gibson (2003) Over- and ectopic expression of Wnt3 causes progressive loss of ameloblasts in postnatal mouse incisor teeth. Connect Tissue Res 44(suppl 1): 124-129.

Nelson, W.J., R. Nusse (2004) Convergence of Wnt, $\beta$-catenin, and cadherin pathways. Science 303: 1483-1487.

O'Gorman, D.M., C.M. Tierney, O. Brennan, F.J. O'Brien (2012) The marine-derived, multimineral formula, Aquamin, enhances mineralisation of osteoblast cells in vitro. Phytother Res 26: 375-380.
Oshima, T., M. Abe, J. Asano, T. Hara, K. Kitazoe, E. Sekimoto, Y. Tanaka, H. Shibata, T. Hashimoto, S. Ozaki, S. Kido, D. Inoue, T. Matsumoto (2005) Myeloma cells suppress bone formation by secreting a soluble Wnt inhibitor, sFRP-2. Blood 106: 3160-3165.

Otsu, K., R. Kishigami, N. Fujiwara, K. Ishizeki, H. Harada (2011) Functional role of Rho-kinase in ameloblast differentiation. J Cell Physiol 226: 2527-2534.

Peng, L., Y. Li, K. Shusterman, M. Kuehl, C.W. Gibson (2011) Wnt-RhoA signaling is involved in dental enamel development. Eur J Oral Sci 119(suppl 1): 41-49.

Qiu, R.G., J. Chen, F. Mccormick, M. Symons (1995) A role for Rho in Ras transformation. Proc Natl Acad Sci USA 92: 11781-11785.

Riksen, E.A., A. Kalvik, S. Brookes, A. Hynne, M.L. Snead, S.P. Lyngstadaas, J.E. Reseland (2011) Fluoride reduces the expression of enamel proteins and cytokines in an ameloblast-derived cell line. Arch Oral Biol 56: 324 330.

Rossol-Allison, J., L.N. Stemmle, K.I. SwensonFields, P. Kelly, P.E. Fields, S.J. McCall, P.J. Casey, T.A. Fields (2009) Rho GTPase activity modulates Wnt3a/ $\beta$-catenin signaling. Cell Signal 21: 1559-1568.

Suomalainen, M., I. Thesleff (2010) Patterns of Wnt pathway activity in the mouse incisor indicate absence of $\mathrm{Wnt} / \beta$-catenin signaling in the epithelial stem cells. Dev Dyn 239: 364372 .

Takahashi, S., N. Kawashima, K. Sakamoto, A Nakata, T. Kameda, T. Sugiyama, K. Katsube, H. Suda (2007) Differentiation of an ameloblast-lineage cell line (ALC) is induced by Sonic hedgehog signaling. Biochem Biophys Res Commun 353: 405-411.

van Aelst, L., M. Symons (2002) Role of Rho family GTPases in epithelial morphogenesis. Genes Dev 16: 1032-1054.

Vincent, S., M. Brouns, M.J. Hart, J. Settleman (1998) Evidence for distinct mechanisms of transition state stabilization of GTPases by fluoride. Proc Natl Acad Sci USA 95: 22102215.

Wong, M.C., J. Clarkson, A.M. Glenny, E.C. Lo, V.C. Marinho, B.W. Tsang, T. Walsh, H.V. Worthington (2011) Cochrane reviews on the benefits/risks of fluoride toothpastes. J Dent Res 90: 573-579.

Zhu, S., L. Liu, V. Korzh, Z. Gong, B.C. Low (2006) RhoA acts downstream of Wnt5 and Wnt11 to regulate convergence and extension movements by involving effectors Rho kinase and Diaphanous: use of zebrafish as an in vivo model for GTPase signaling. Cell Signal 18: 359-372. 